Original paper

\title{
Radiochromic films for dental CT dosimetry: A feasibility study
}

\author{
O. Rampado ${ }^{\mathrm{a}, *}$, S.D. Bianchi ${ }^{\mathrm{b}, 1}$, A. Peruzzo Cornetto $^{\mathrm{a}, 2}$, V. Rossetti $^{\mathrm{a}, 2}$, R. Ropolo ${ }^{\mathrm{a}, 2}$ \\ ${ }^{a}$ S.C. Fisica Sanitaria, A.O.U. San Giovanni Battista di Torino, Corso Bramante 88, 10126 Torino, Italy \\ ${ }^{\mathrm{b}}$ Dipartimento Discipline Medico Chirurgiche, Università degli Studi di Torino, Via Genova 3, 10126 Torino, Italy
}

\section{A R T I C L E I N F O}

\section{Article history:}

Received 11 January 2012

Received in revised form

31 May 2012

Accepted 13 June 2012

Available online 30 June 2012

\section{Keywords:}

Patient dose

Radiochromic film

Dentomaxillofacial

Cone beam CT

Multislice CT

\begin{abstract}
A B S T R A C T
Dental CT dose evaluations are commonly performed using thermoluminescent dosimeters (TLD) inside anthropomorphic phantoms. Radiochromic films with good sensitivity in the X-ray diagnostic field have recently been developed and are commercially available as GAFCHROMIC XR-QA. There are potential advantages in the use of radiochromic films such as a more comprehensive dosimetry thanks to the adjustable size of the film samples. The purpose of this study was to investigate the feasibility of using radiochromic films for dental CT dose evaluations.

Film samples were cut with a width of $5 \mathrm{~mm}$ and a length of $25 \mathrm{~mm}$ (strips), the same size as the Alderson Rando anthropomorphic phantom holes used in this study. Dental CT dose measurements were performed using simultaneously both TLD and radiochromic strips in the same phantom sites. Two equipment types were considered for dental CT examinations: a 16 slice CT and a cone beam CT. Organ equivalent doses were then obtained averaging the measurements from the sites of the same organ and effective doses were calculated using ICRP 103 weighting factors. The entire procedure was repeated four times for each CT in order to compare also the repeatability of the two dosimeter types.

A linear correlation was found between the absorbed dose evaluated with radiochromic films and with TLD, with slopes of 0.930 and 0.944 (correlation $r>0.99$ ). The maximum difference between the two dosimeter's measurements was $25 \%$, whereas the average difference was $7 \%$. The measurement repeatability was comparable for the two dosimeters at cumulative doses above $15 \mathrm{mGy}$ (estimated uncertainty at 1 sigma level of about 5\%), whereas below this threshold radiochromic films show a greater dispersion of data, of about $10 \%$ at 1 sigma level. We obtained, using respectively Gafchromic and TLD measurements, effective dose values of $107 \mu \mathrm{Sv}$ and $117 \mu \mathrm{Sv}$ (i.e. difference of $8.6 \%$ ) for the cone beam CT and of $523 \mu \mathrm{Sv}$ and $562 \mu \mathrm{Sv}$ (i.e. difference of $7 \%$ ) for the multislice $\mathrm{CT}$.

This study demonstrates the feasibility of radiochromic films for dental CT dosimetry, pointing out a good agreement with the results obtained using TLD, with potential advantages and the chance of a more extensive dose investigation.
\end{abstract}

(c) 2012 Associazione Italiana di Fisica Medica. Published by Elsevier Ltd. All rights reserved.

\section{Introduction}

The use of three dimensional information in dentomaxillofacial radiology and surgery planning has consistently grown over the last few years, firstly as a consequence of a more extensive use of Multislice CT (MSCT) combined with dedicated reformatting software (dentascan) and, more recently, due to the development and diffusion of several pieces of Cone Beam CT (CBCT) equipment.

\footnotetext{
* Corresponding author. Tel.: +39 0 116335373; fax: +39 0116634547. E-mail addresses: orampado@molinette.piemonte.it (O. Rampado), silviodiego.bianchi@unito.i (S.D. Bianchi), andrea-peruzzo@libero.it (A. Peruzzo Cornetto), vrossetti@molinette.piemonte.it (V. Rossetti), rropolo@ molinette.piemonte.it (R. Ropolo).

1 Tel.: +390 116965183; fax: +390116960310.

2 Tel.: +390 116335373; fax: +390 116634547 .
}

Patient radiation dose is one of the main issues in dental CT examinations and was investigated in several studies [1-9]. Although the related effective dose is lower than most other CT examinations involving thorax and abdomen anatomical districts, there is concern about radiation dose in dental CT as a consequence of the frequency of dental examinations and of the repetition on the same subject, especially for paediatric patients. Therefore it is important to know dental CT patient dose for all machines and protocols, in order to optimise acquisition parameters and to minimise the related radiological risk. Several studies compare the MSCT patient dose with the CBCT patient dose [7-9]. Generally the results show doses for MSCT equipment ranging from two to ten times the CBCT doses, but it must be underlined that there is a great variability in the MSCT protocol definition and often there are possibilities of optimisation which have been not completely investigated $[10,11]$. 
The most significant dosimetric quantity with regards to dental CT patient dose is the effective dose which, as reported in ICRP 103 [12], is a suitable quantity with which to compare different radiological techniques and equipment for the same type of examination. Other dose indexes, like volumetric Computed Tomography Dose Index (CTDIvol) and Dose Length Product (DLP), can be used for optimisation studies with MSCT, but there are several concerns about their use in $\mathrm{CBCT}$ due to the particular exposure geometry and the consequent dose distribution [5]. Effective dose is usually evaluated by means of thermoluminescent dosimeters (TLD) positioned in an anthropomorphic phantom. TLD dosimetry is timeconsuming and requires particular care in calibration, dosimeter batch selection and measurement procedures. In order to correctly evaluate the organ dose distribution with a partial irradiation it is also important to have a sufficient number of dosimeters involved.

Radiochromic films with good sensitivity in the X-ray diagnostic field have been recently developed and are commercially available as GAFCHROMIC XR-QA and XR-CT (International Specialty Products, Wayne, NJ). Several studies $[13,14]$ investigated the dose and energy dependence of these films, showing a dynamic dose range from 1 to $100 \mathrm{mGy}$ and a film maximum relative response in the 50-70 keV equivalent photon energy range. There are potential advantages in the use of radiochromic films such as the possibility of a more comprehensive dosimetry thanks to the adjustable size of the film samples.

The purpose of this study was to investigate the feasibility of using radiochromic films for dental CT dose evaluations, comparing the obtainable dosimetric results with those of TLD dosimeters.

\section{Material and methods}

The anthropomorphic phantom Alderson Rando (The Phantom Laboratory, New York, USA) was used in this study in order to evaluate absorbed organ doses using both types of dosimeters. This phantom is manufactured with a natural human skeleton embedded in a proprietary urethane formulation with an effective atomic number and mass density that closely simulates muscle tissue with randomly distributed fat. The head and neck part is sliced into eleven $2.5 \mathrm{~cm}$ thick sections, with several holes of $5 \mathrm{~mm}$ diameter.

The TLDs used were rods measuring $1 \times 1 \times 6 \mathrm{~mm}^{3}$, made of lithium fluoride (LiF:Mg,Ti), TLD-100 from Harshaw, Ohio. In order to select homogeneous batches of TLDs with a reproducible response, the procedure of irradiation and reading was repeated five times for all rods with the same dose.

Three different batches with homogeneous dose response were identified and all rods that deviated more than 5\% (one standard deviation) from each batch mean were rejected. The number of TLDs finally selected for each batch was 50 . TLDs were read using a Harshaw 3500 manual TLD reader controlled by a PC-based, online, menu driven by TLDshell software (Bicron-Harshaw). The glow curve of each TLD reading was displayed by the software. The readout cycle consisted of a preheat to $100^{\circ} \mathrm{C}$ for $10 \mathrm{~s}$, followed by a subsequent heating at $6^{\circ} \mathrm{C} / \mathrm{s}$ to $260^{\circ} \mathrm{C}$ and then keeping this temperature of $260^{\circ} \mathrm{C}$ constant for another $10 \mathrm{~s}$. The cycle was chosen after considering [15] and after visual examination of glow curve from a number of different heating times and maximum temperature. The total readout time was about $47 \mathrm{~s}$. Nitrogen gas was used to remove oxygen from the sample compartment. The annealing procedure was the standard one used in our department, a common one for $\mathrm{LiF}(\mathrm{Mg}, \mathrm{Ti})$, and consisted in a first annealing at $400{ }^{\circ} \mathrm{C}$ for $1 \mathrm{~h}$, followed by a second one at $100^{\circ} \mathrm{C}$ for $2 \mathrm{~h}$. Each time the rods were removed from the oven, they were rapidly cooled on a large brass block. After irradiation, but before readout, the TLDs were annealed for $10 \mathrm{~min}$ at $100{ }^{\circ} \mathrm{C}$ to clear the low energy traps, as suggested by Ref. [16].

The radiochromic film model XR-QA, lot number 48022-09A, was used in this study. Film samples were cut with a width of $5 \mathrm{~mm}$ and a length of $25 \mathrm{~mm}$, the same size as the phantom holes. Exposed films were digitised with an Epson Expression $10000 \mathrm{XL}$ (Epson Seiko Corporation, Nagano, Japan), by setting the following scanning parameters: RGB mode (16 bit per colour), $75 \mathrm{dpi}$, no colour correction factors or filters. The red components of the images, being more sensitive to radiation [17], were used for analysis.

Details of the approach used for the scanner characterisation and for the scanning procedure are provided in another paper [13] and here only briefly summarised. The spatial uniformity of the scanner was tested and a rectangular area with uniform response was identified and delineated with an opaque mask on the scanner window in order to use only this area for digitisation. Also the scanner response reproducibility was evaluated and the relative contribution to the total uncertainty was taken into consideration. An image printed on photographic paper with 50 holes of the same dimensions as the film strips was used as position mask.

To avoid the effect of occasional instabilities of the scanner, an automatic check procedure was implemented and applied to each scanned image to verify that the average pixel values of the scale calibration tablet indicated in Fig. 1(a) differ from the expected values by less than two times the standard deviations previously determined. A Kodak grey scale calibration tablet was also present in the acquisition area, as suggested in Ref. [18]. Furthermore, as suggested in Ref. [19], five images were acquired and averaged for every measurement.

The images obtained from film digitalisation were analysed using ImageJ (Wayne Rusband, National Institutes of Health, MD, http://rsb.info.nih.gov/ij/) with plug-ins written in-house. Every film sample was scanned before and after the exposure in the same scanner position. The five scans of the unexposed films and the five scans of the exposed films were averaged, and a new image with values for each pixel calculated as

net $\Delta \mathrm{PV}=k \frac{\mathrm{PV} \text { unexp }}{\mathrm{PV}_{\mathrm{exp}}}$

was created, where $P V_{\text {unexp }}$ was the pixel value of the unexposed films image, $P V_{\text {exp }}$ was the pixel value in the same position as the exposed films image, and $k$ is an arbitrary multiplying factor. The maximum value of the quantity $P V_{\text {unexp }} / \mathrm{PV}_{\text {exp }}$, was in the range from $1(0 \mathrm{mGy})$ to about 3 (100 $\mathrm{mGy}$ ). The value of the multiplying factor $k$ defines the range of values for the net $\Delta P V$ quantity, which, being a pixel value, must be integer and less than $2^{16}=65,536$. As a consequence, a $k$ value of 20,000 was chosen.

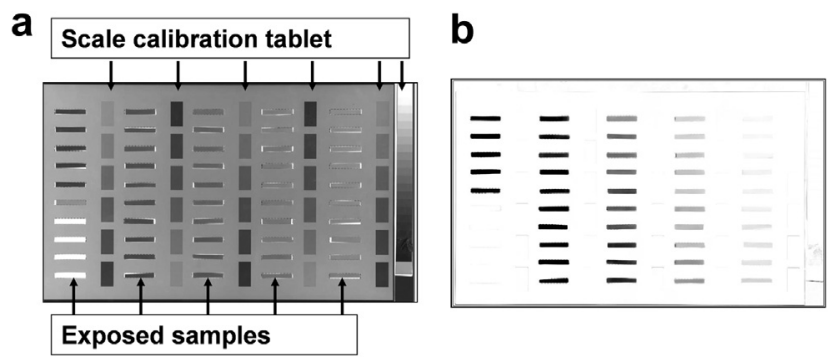

Figure 1. Red channel images of film samples (a) exposed during the calibration procedure. An image printed on photographic paper with 50 holes of the same dimensions as the film strips was used as position mask, with several grey scale calibration tablets used to check the scanner response stability. (b) Shows the dose image of film samples, resulted from the ratio between the image of unexposed films and the image of exposed films, and the application of the calibration curve. 
A single net $\triangle \mathrm{PV}$ region of interest (ROI) value was calculated for every sample of the calibration set, averaging the pixel values in an ROI measuring $1 \mathrm{~mm} \times 2 \mathrm{~cm}$, together with the ROI standard deviation. Delivered doses were plotted as a function of the measured net $\Delta \mathrm{PV}$ and a suitable fit function was searched for minimising both the number of fitting parameters and their relative uncertainty. The fits were performed using a tool from the MATLAB 6.5 software (The Mathworks, Natick, MA) with the "LevenbergMarquardt" minimisation method. For the film lot used in this study, we found a good calibration curve using a simple second order polynomial function

$D_{\text {fit }}=b(\text { net } \Delta \mathrm{PV}-k)^{2}+c($ net $\Delta \mathrm{PV}-k)$

Also in this relationship the parameter $k$ is the same as the one in Eq. (1).

Figure 1(b) shows the dose image of film samples, resulting from the ratio between the unexposed and the exposed films image, corrected by the application of the dose calibration curve. The calibration curve obtained for the beam with the MSCT characteristics is shown in Fig. 2.

The uncertainty in the measurement of an unknown dose was estimated using the Gaussian propagation of errors and assuming the absence of cross-correlation terms. A detailed description of the dose uncertainty analysis with this kind of radiochromic dosimetry has been reported in Ref. [13] and here only briefly summarised. A dose value evaluated with the expression (2) will be affected by an uncertainty defined by the following relationship:

$\sigma_{\mathrm{D}}^{2}=\left(\frac{\partial D_{\mathrm{fit}}}{\partial b}\right)^{2} \sigma_{b}^{2}+\left(\frac{\partial D_{\mathrm{fit}}}{\partial c}\right)^{2} \sigma_{c}^{2}+\left(\frac{\partial D_{\mathrm{fit}}}{\partial \text { net } \Delta \mathrm{PV}}\right)^{2} \sigma_{\text {net } \Delta \mathrm{PV}}^{2}$

where $\sigma_{\mathrm{D}}$ is the total estimated uncertainty (at 1 sigma level) for a dose determined using Eq. (2), $\sigma_{b}$ and $\sigma_{c}$ represent the standard deviations for the fitting parameters $b$ and $c$ provided by the fitting calculation software and $\sigma_{\text {net } \Delta \mathrm{PV}}$ the uncertainty for the net $\Delta \mathrm{PV}$ quantity.

The method for the calculation of $\sigma_{\text {net } \Delta \mathrm{PV}}$ was described in Ref. [13] and the approach used considered three different contributions for its quantification: the standard deviation of the ROIs $\sigma_{\mathrm{ROInet} \Delta \mathrm{PV}}$ related to the scanner and film response noise, a contribution related to the scanner response repeatability $\sigma_{\text {scanner }}$ and a contribution due to the film response uniformity $\sigma_{\text {film }}$ calculated considering the net $\Delta \mathrm{PV}$ differences for film samples exposed to the same dose. The summation in quadrature of these contributions gives the actual uncertainty:

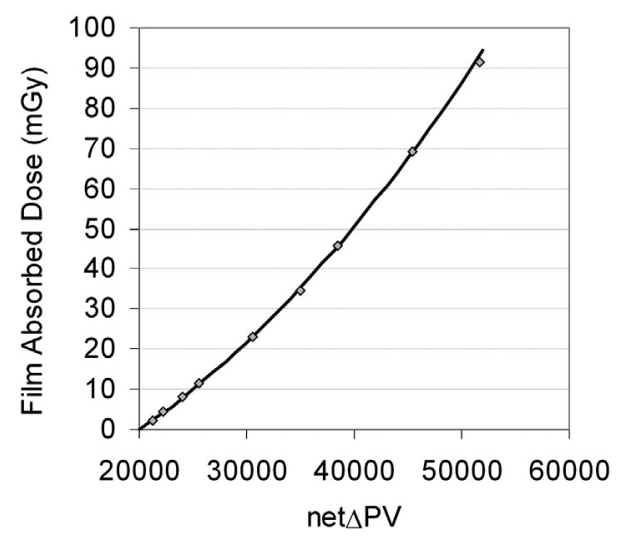

Figure 2. Calibration curve for GafChromic XR-QA film model and for the beam with the MSCT characteristics. $\sigma_{\text {net } \Delta \mathrm{PV}}=\sqrt{\left(\sigma_{\mathrm{ROInet} \Delta \mathrm{PV}}\right)^{2}+\left(\sigma_{\text {scanner }}\right)^{2}+\left(\sigma_{\text {film }}\right)^{2}}$

The following percentage uncertainty values were obtained for the film samples and scanner used in this study: $0.35 \%$ for $\sigma_{\text {ROI- }}$ net $\Delta \mathrm{PV}, 0.45 \%$ for $\sigma_{\text {scanner }}$ and $0.4 \%$ for $\sigma_{\text {film. }}$. The total dose uncertainty resulted to be less than $5 \%$ for doses above $5 \mathrm{mGy}$ and of the order of $15 \%$ for doses of about $1 \mathrm{mGy}$.

Both film samples and TLDs were calibrated by simultaneous irradiation with a conventional X-ray tube, with a selection of the beam energy and of the added filtration in order to match the beam characteristics of the MSCT $(120 \mathrm{kVp}$ with $5 \mathrm{~mm}$ of added $\mathrm{Al}$ filtration) and of the $\mathrm{CBCT}(110 \mathrm{kVp}$ with $12 \mathrm{~mm}$ of added $\mathrm{Al}$ filtration) used in this study. For each calibration point, 12 TLD rods ( 4 for each batch) and 4 film samples were exposed to a homogeneous beam with simultaneous measurements of dose performed by an ionisation chamber (type $25710.6 \mathrm{cc}$, NE technology limited, Berkshire, England; electrometer model UNIDOS, PTW, Freiburg, Germany), positioned on a Styrofoam block in order to have a negligible contribution of backscatter radiation. The ionisation chamber was previously calibrated by a standard dosimetry laboratory in terms of Air-Kerma in air, with exposure in free air to a beam of $100 \mathrm{kV}$ filtered with $4 \mathrm{~mm} \mathrm{Al}$. The formalism employed to convert Air-Kerma in air to dose is based on TG-61 protocol [20], as also described in Refs. [21,22], using the following equation:

$D=\left(K_{\mathrm{air}}^{\mathrm{film}}\right) \cdot\left(\frac{\mu_{\mathrm{en}}}{\rho}\right)_{\mathrm{air}}^{\mathrm{w}}$

where $\left(\mu_{\mathrm{en}} / \rho\right)_{\text {air }}^{\mathrm{w}}$ is in-air mass energy-absorption coefficient ratio, water-to-air, which is tabulated in the TG-61 protocol as a function of HVLs.

Dental CT dose measurements were performed using both TLD and radiochromic strips simultaneously positioned in the same sites of the anthropomorphic phantom. 47 locations and different organs were investigated with reference to the following distribution: bone marrow and or surface 23 , brain 7 , thyroid 6 , salivary glands 6, oesophagus 1 and other remainder tissues 4 (oral mucosa, extrathoracic airways, lymph nodes). Figure 3 shows the phantom with indication of used sites. In order to consider the most significant position for organ dose assessment the TLDs were positioned sometimes in the central part of the hole (at the middle of the slice thickness), and in other cases in the cranial or caudal part as indicated in the figure. The corresponding film dose values were assessed positioning an ROI on the film image at the same relative position along the sample length. Organ equivalent doses were then obtained averaging the measurements from the sites of the same organ, considering the mass fractions per phantom slice published [23] about bone marrow, bone surface and thyroid. Location and mass fractions for salivary glands, brain, oesophagus, oral mucosa, extrathoracic airways, lymph nodes and muscle were derived considering anatomical atlas and data provided by ICRP [24]. The skin was not considered in this study, because a large number of additional dosimeters should have been employed to have a consistent evaluation of average absorbed dose and at the same time a minor contribution to the effective dose was expected, of the order of $1 \%$ according to Ref. [1].

MSCT dose measurements were performed with a 16 slice CT scanner (Lightspeed 16Pro, GE, Milwaukee, Wisconsin, USA). The acquisition protocol parameters used were: $120 \mathrm{kV}, 150 \mathrm{~mA}, 1 \mathrm{~s}$ for rotation, slice thickness $0.625 \mathrm{~mm}$, pitch 0.562 , field of view $128 \mathrm{~mm}$. The high value of $\mathrm{mA}$ was set in order to have the optimal response of both dosimeters, providing absorbed doses greater than $1 \mathrm{mGy}$ to all sites in order to substantially exceed the background dose values. The volume scan length of the MSCT was 

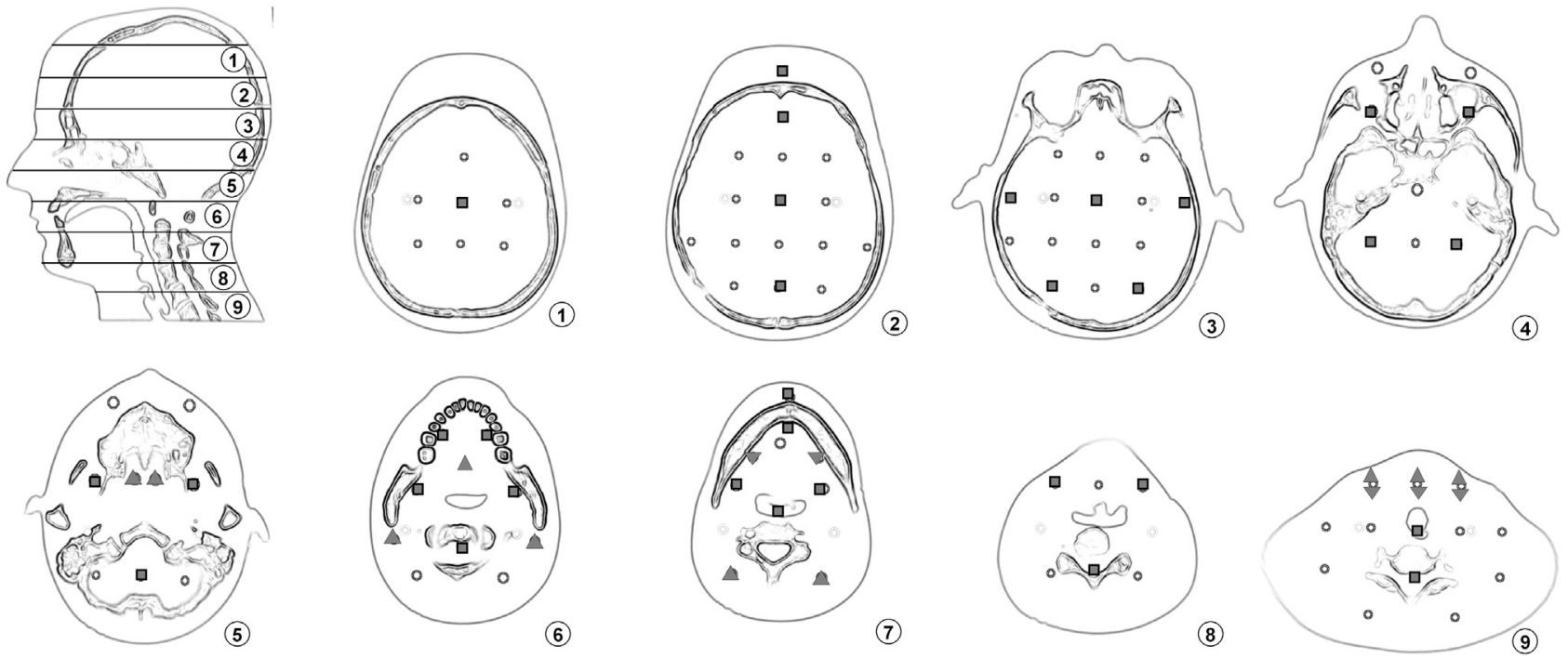

Position of measurement points in the hole:

$\Delta$ Cranial part

- Central part $\nabla$ Caudal part

Figure 3. Dosimeter locations inside the anthropomorphic phantom.

$8.1 \mathrm{~cm}$, that is the minimum selectable in order to obtain a complete maxillofacial scan of the phantom.

CBCT dose measurements were performed with a Newtom VG 3D (QR SRL, Verona, Italy). Acquisition protocol parameters were automatically set by the equipment as follow: $110 \mathrm{kV}, 2.2 \mathrm{~mA}, 18 \mathrm{~s}$ for rotation with $3.6 \mathrm{~s}$ effective exposure time, field of view with a diameter of $150 \mathrm{~mm}$ and a height of $10.5 \mathrm{~mm}$. Ten acquisitions were performed with the same dosimeters group in order to achieve, for this equipment also, cumulative dose values per single site above $1 \mathrm{mGy}$. For both machines the entire exposure procedure was repeated four times in order to also compare the repeatability of the two dosimeters type.

\section{Results}

Figure 4 shows the comparison between the absorbed dose evaluated with radiochromic films and with TLD, considering the average values of the four performed measurements. A good linear correlation was found between the absorbed dose evaluated with radiochromic films and with TLD for both radiological machines, with a slope of 0.930 (correlation $r=0.998$ ) for the CBCT and a slope of 0.944 (correlation $r=0.997$ ) for the MSCT. Linear regressions were calculated forcing the intercept to zero, as both measurement methods involved a background subtraction and, as a consequence, a null dose for radiochromic films was expected when a null dose was measured with TLD. The maximum difference between the two dosimeters' measurements was $25 \%$, whereas the average difference was of about $7 \%$.

Figure 5 shows the percentage differences of single measurement values from the average measurement for each dosimeter site and for the different exposures. The graph highlights the repeatability of measurements for both dosimeters and equipment. For radiochromic films and $\mathrm{CBCT}$ equipment (Fig. 5(a)), higher percentage differences were observed for lower doses. Below the threshold of $15 \mathrm{mGy}$, the average difference was 7\% and about 95\% of measurements showed differences below 20\%. Above $15 \mathrm{mGy}$, the average difference was 3.5\% and 95\% of measurements have differences below 10\%. A different behaviour was observed for TLD measurements, with substantially the same data dispersion over all the measurement range. The average percentage difference was 3\% and about $95 \%$ of measurements showed differences below $9 \%$. For MSCT equipment (Fig. 5(b)), the graph shows two separate data patterns: the first refers to dose values below $20 \mathrm{mGy}$ and contains absorbed dose data from scattered radiation, whereas the second (which refers to doses above $25 \mathrm{mGy}$ ) contains absorbed dose data from the primary X-ray beam. For radiochromic film
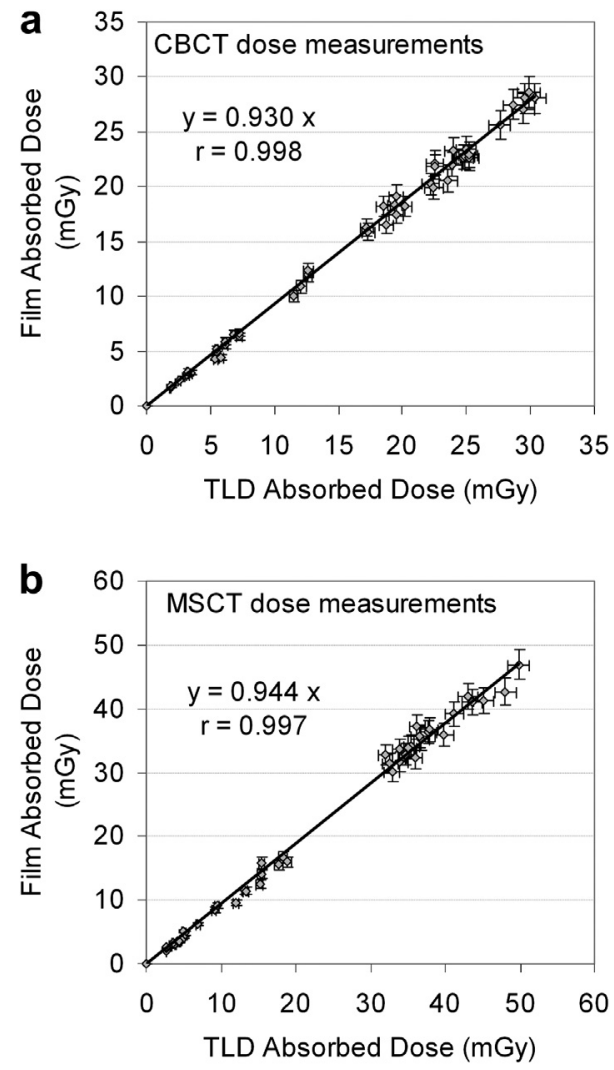

Figure 4. Comparison of the absorbed dose measurements with radiochromic film samples and with TLD, for the CBCT (a) and for the MSCT (b). A linear correlation was found between the two quantities for both machines. 

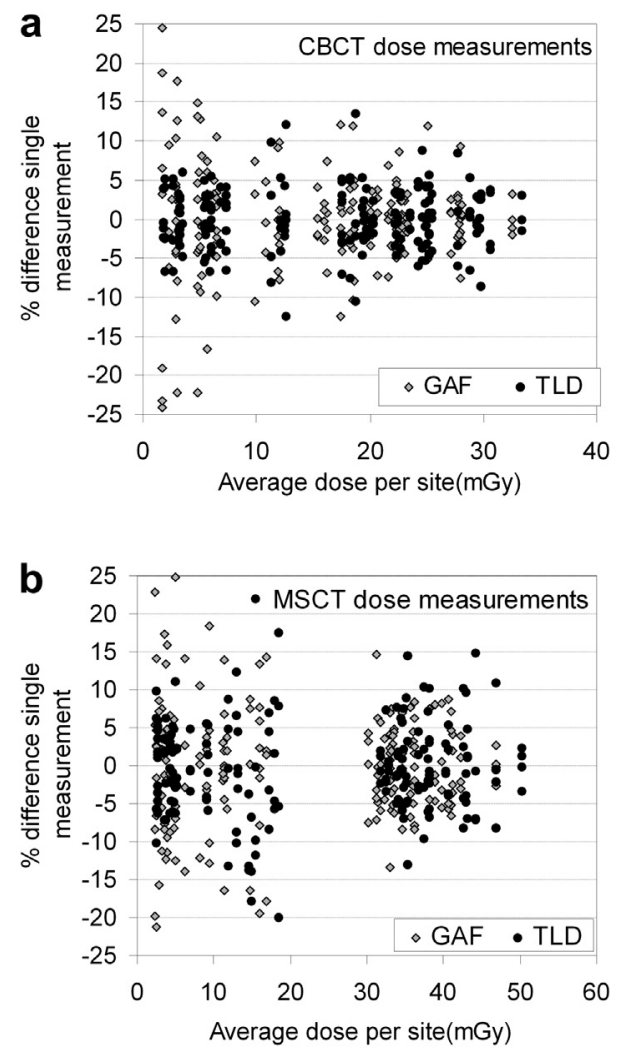

Figure 5. Percentage differences between the single measurements and the average measurement for each site and for all the exposures performed in this study, with the CBCT (a) and with the MSCT (b) equipment.

measurements, the average percentage difference for doses below $20 \mathrm{mGy}$ was $7.2 \%$ and about $95 \%$ of measurements showed differences below $20 \%$. Above $25 \mathrm{mGy}$, the average difference was $4.0 \%$ and about $95 \%$ of measurements showed differences below $10 \%$. TLD measurements show also for the MSCT an almost constant dispersion over all the measurement range, with an average difference of $5 \%$ and $95 \%$ of measurements with differences below $12 \%$.

Organ average absorbed doses and effective doses for the two machines and for the two dosimeters types are shown in Table 1. For the CBCT, dose values are calculated for a single acquisition (one tenth of the measured doses obtained with ten repeated acquisitions). For the MSCT, dose values are normalised to a tube current times rotation exposure time of $60 \mathrm{~mA}$, that is the value used in the reference protocol of our institution. The percentage differences between the organ doses carried out with the two different types of

Table 1

Equivalent and effective doses for the two machines and for the two dosimeters types.

\begin{tabular}{|c|c|c|c|c|c|c|}
\hline \multirow[t]{2}{*}{ Organ } & \multicolumn{3}{|l|}{$\mathrm{CBCT}$} & \multicolumn{3}{|l|}{ MSCT } \\
\hline & $\begin{array}{l}\text { Film dose } \\
(\mu \mathrm{Sv})\end{array}$ & $\begin{array}{l}\text { TLD dose } \\
(\mu \mathrm{Sv})\end{array}$ & \% Differ. & $\begin{array}{l}\text { Film dose } \\
(\mu \mathrm{Sv})\end{array}$ & $\begin{array}{l}\text { TLD dose } \\
(\mu \mathrm{Sv})\end{array}$ & \% Differ. \\
\hline Bone marrow & 157 & 173 & -9.1 & 1017 & 1075 & -5.4 \\
\hline Bone surface & 726 & 801 & -9.3 & 4723 & 4989 & -5.3 \\
\hline Salivary glands & 2474 & 2705 & -8.5 & 10,949 & 11,973 & -8.5 \\
\hline Brain & 639 & 724 & -11.7 & 6153 & 6599 & -6.8 \\
\hline Thyroid & 484 & 534 & -9.3 & 751 & 839 & -10.5 \\
\hline Oesophagus & 56 & 60 & -7.0 & 96 & 106 & -9.2 \\
\hline Remainder & 232 & 251 & -7.2 & 1240 & 1333 & -6.9 \\
\hline Effective dose & 107 & 117 & -8.6 & 523 & 562 & -7.0 \\
\hline
\end{tabular}

dosimeters are in agreement with the single measurement differences herein reported.

\section{Discussion}

The comparison of radiochromic and TLD absorbed dose measurements in anthropomorphic phantom performed in this study was aimed to assess the relative accuracy and precision of the two dosimeter types. Moreover, the potential advantages of radiochromic films were investigated considering practical aspects and recorded dose distribution information.

The obtained results for both the machines used showed how the absorbed doses measured with radiochromic film were smaller than those measured with TLD, pointing out a difference of about $7 \%$. The reason for this difference was not investigated in this work, but some indications are deducible from previous published studies. One contribution to this difference is related to the angular dependence of film samples with the used size, investigated in Ref. [25]. The difference between the dose response with the X-ray beam perpendicular to the film surface $\left(0^{\circ}\right)$ and the dose response at angles in the range $0^{\circ}-70^{\circ}$ was $<1 \%$. The same behaviour was found for the exposures of the film strips on the opposite side, for angles in the range $110^{\circ}-180^{\circ}$. At $80^{\circ}$ and at $100^{\circ}$, a medium difference of $3 \%$ was found. The stronger decrease in dose response was observed between $85^{\circ}$ and $95^{\circ}$. The average dose response at $85^{\circ}$ and at $95^{\circ}$ was $91 \%$ of the dose response at $0^{\circ}$, whereas at $90^{\circ}$ (film surface parallel to the axis beam) the relative response was about $49 \%$. For an axial scan in computed tomography, the cumulative dose response of the film can be considered as a sum of the single contributions at different angles and, integrating over the range $0^{\circ}-360^{\circ}$, a relative response decrease of $2 \%$ was obtained. This behaviour is different for the TLD, where an isotropic response is expected.

Another possible reason for the differences could be found in the different energy responses of the two dosimeters. The calibration was performed for both dosimeters with a beam quality similar to that of the CT radiation beam, with dosimeters positioned in air on a Styrofoam block. This is a calibration set-up usually employed for dosimetry in diagnostic radiology, considering the energy response variation not relevant and within the required accuracy of $7 \%$ [26]. The influence of the TLDs energy dependence for organ dose assessment was investigated by Struelens et al. [27] comparing TLD measurements and Monte Carlo simulations. They calculated the energy spectra within the phantom inside and outside the radiation field for an abdomen PA projection. The comparison to the source energy spectrum showed effective energies to be 10-15\% lower, with a peak in the energy spectrum between 25 and $30 \mathrm{keV}$ that corresponds to the fluorescence peak of antimony ( $\mathrm{Sb}$ ), which is present in a small fraction in the softtissue material of the Rando-Alderson phantom. With respect to the energy of the X-ray source, they found a relative response of the TLDs to be between $2.0 \%$ and $3.5 \%$ higher. Other published studies $[13,14,28]$ investigated the energy dependence of TLDs and of radiochromic XR-QA and XR-CT films. Figure 6 shows the relative response of the dosimeters extracted from this literature, normalised to the response at an effective energy of $63 \mathrm{keV}$ that is close to that of the beams used in this study (62 keV for the MSCT beam and $64 \mathrm{keV}$ for the CBCT beam). The TLD relative response is greater than 1 for effective energies in the range $20-50 \mathrm{keV}$, whereas the film relative response in this energy range is below 1 for both the cited studies. This different response may have contributed to the differences in the dose evaluations obtained in this study. Further investigations are needed to analyse the different energy responses of the two dosimeters inside the phantom and to optimise the calibration procedure in order to improve the accuracy, for example 


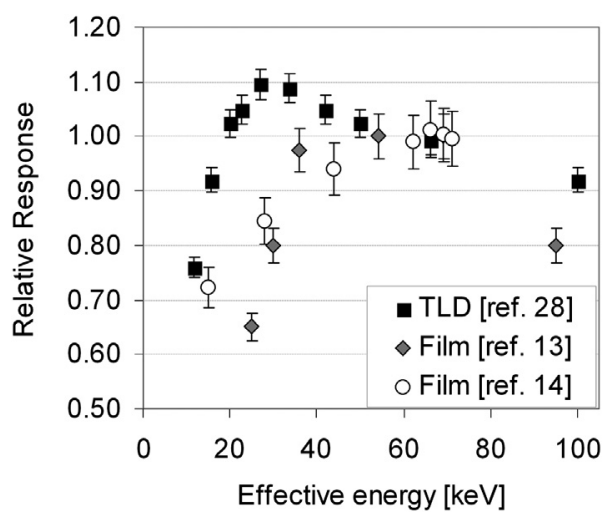

Figure 6. Dosimeters relative response extracted from the literature, normalised to the response at an effective energy of $63 \mathrm{keV}$ that is close to that of the beams used in this study (62 keV for the MSCT beam and $64 \mathrm{keV}$ for the CBCT beam).

placing the dosimeters between two layers of PMMA or using a water phantom.

The repeatability of measurements was better for TLD dosimeters over all the measurement range. In the Results section, the percentage difference between single measurements and average measurements per site was investigated. Threshold values were indicated which were not exceeded by $95 \%$ of measurements, for both machines and dosimeters. In other words, these thresholds can be interpreted as a $2 \sigma$ level of uncertainty, in agreement with the Gaussian distribution. On this basis, a $1 \sigma$ uncertainty of $10 \%$ was estimated for radiochromic films for doses below a threshold of $15 \mathrm{mGy}$ for CBCT and of $20 \mathrm{mGy}$ for MSCT, whereas a 5\% value was estimated for higher doses. TLD measurements show an almost constant $1 \sigma$ uncertainty in the dose range investigated, with a value of about $4.5 \%$ for CBCT and $6 \%$ for MSCT. The main error sources for TLD measurements are related to the reading process, the position repeatability and the exposure intensity constancy, but the same rod was used in each site for all the exposures. For the radiochromic films, different film samples were cut for each exposure, and the homogeneity of film influences the response repeatability. Moreover, film darkening below $10 \mathrm{mGy}$ is very weak and as a consequence the scanner uncertainties have a greater influence on the final extracted dose value. The uncertainty of low dose measurements could be partially reduced by increasing the cumulative exposure and this should be considered for further studies.

It is also interesting to observe that measurement uncertainties were slightly higher for MSCT measurements. This is probably related to the geometrical exposure condition in volumetric MSCT and to the repeatability in the phantom position. As outlined in several studies [29,30], in helical multidetector computed tomography, there are significant variations of $z$-axis doses at phantom surfaces. Doses within those regions where direct primary beam exposure overlap occurs during helical scanning are greater and doses to regions where "gaps" in the primary beam exposure occur are lower. Whether primary beam exposure overlaps or gaps occur depends on pitch, nominal and actual beam widths, beam divergence, and the selected acquisition volume and phantom position. At any moment in time during a helical scan, regions within a phantom that are closer to the X-ray tube position will have a higher dose rate than those regions which are farther away. Resulting cumulative dose distributions at peripheral locations due to helical scanning are locally periodic in space with a fundamental period equal to the table translation per rotation, which will depend on pitch and detector collimation width. During the spiral acquisition, for all the dosimeters located close to the phantom surface the greatest dose contribution is provided by the beam angular positions with lowest crossed phantom thickness. As a consequence, an uncertainty of only a few millimetres in the phantom position can significantly change the relative positions of the spiral exposure and thus the dose of the single dosimeters sites. This effect is not expected for CBCT measurements, as the cumulative dose distribution at peripheral locations has a profile with almost uniform dose in the primary beam and tails of scattered dose outside. As a consequence the phantom position uncertainty will heavily affect only the dose measurement sites at the boundaries of the primary beam.

The obtained effective dose values for CBCT and MSCT can be compared with those published in other studies. Several papers investigated the effective dose for Newtom CBCT machines, but we found only two works with the model used in this study, the Newtom VG. Davies-Ludlow et al. [31] obtained a value of $109 \mu \mathrm{Sv}$, whereas Pawels et al. [1] published a value of $83 \mu \mathrm{Sv}$. The values of $107 \mu \mathrm{Sv}$ for radiochromic films and of $117 \mu \mathrm{Sv}$ for TLDs obtained in this study are comparable to values published by Davies-Ludlow. Published dose values for MSCT span over a wider range of several hundreds of $\mu \mathrm{Sv}$, as a consequence of the variability in the acquisition parameter protocols. In a study of Suomalainen et al. [32] effective doses were evaluated for several protocols of a GE Lightspeed MSCT similar to the one used in this study. In order to make a comparison, the ratio between the effective dose and the DLP can be considered. This ratio had values in the range $0.0026-$ $0.0030 \mathrm{mSv} /(\mathrm{mGycm})$ for the Suomalainen study, whereas in this work values of 0.0031 for films and 0.0033 for TLDs were obtained.

To our knowledge, there is only one study in literature that investigates the differences between radiochromic films and TLD in CT dose evaluations [33]. In that work, Brady et al. present dose distributions for the lungs, liver and kidneys of a paediatric anthropomorphic phantom, extracted from radiochromic films positioned between the phantom slices. In those exposure conditions they also found a lower dose response for the films, with percentage differences for the different phantom positions in a range from $15 \%$ to $28 \%$. The lower dose evaluation of the film was attributed to the lower detection sensitivity of an X-ray beam when the film is oriented with its large surface area is parallel to the X-ray beam's main axes. Tissue specific correction factors were proposed.

There are potential advantages in the use of radiochromic films for this kind of dosimetry. By focussing on the reading process, the digitalisation procedure for a set of 47 film samples required only a few minutes, whereas approximately $1 \mathrm{~min}$ was necessary for reading each TLD (total time $47 \mathrm{~min}$ ) with our manual reader. The extraction of dose measurements from the film scanned image is performable automatically by an appropriate software procedure, so that the measurement time is not proportional to the number of sites investigated as it is for TLD dosimetry. With a TLD automatic reader, the reading time would probably be comparable to the time needed to read and process film measures.

The main advantage of films is that each sample allowed the dose distribution to be investigated over the entire phantom slice thickness of $25 \mathrm{~mm}$, whereas the TLD is only $6 \mathrm{~mm}$ long. Analysing the film dose distributions, several single strips show a dose gradient resulting from the exposure at edges of the FOV, as shown in the examples in Fig. 7. Considering the different exposure geometrical condition of different $\mathrm{CBCT}$ devices and protocols (different field of view), recent studies [1] pointed out that the only way to precisely estimate the absorbed dose for any organ is to use dosimeters at as many locations as possible, because the absorbed dose is an average dose. It has been demonstrated that organ dose estimations using a low number of dosimeters can deviate $18-28 \%$ with differences up to $80 \%$ [1]. Film strips have the potential to easily increase the dose sampling in the phantom by considering the dose distribution over all the entire area. 

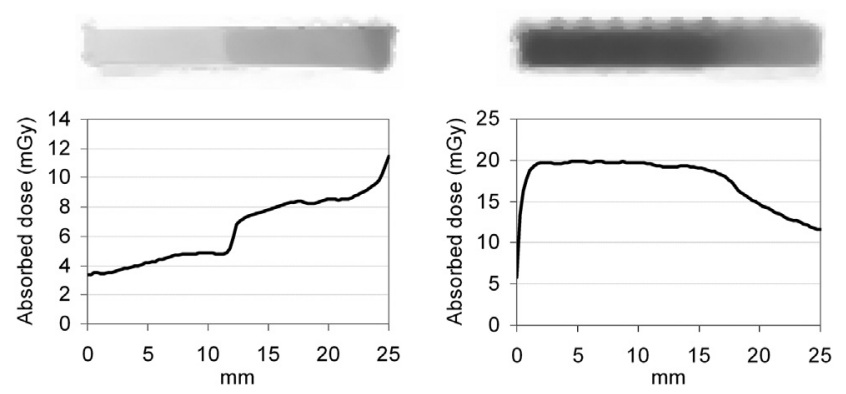

Figure 7. Examples of radiochromic images and dose profiles of dose gradients resulting from the exposure at the boundaries of the primary beam.

Moreover, in terms of economical costs, a TLD dosimetric system (automatic reader and TLD dosimeters) is much more expensive (about ten times) than a scanner with several film lots.

In conclusion, this study demonstrates the feasibility of the use of radiochromic films for dental CT dosimetry, showing results similar to those achievable with TLD, highlighting the possibility of a more extensive dosimetric investigation.

\section{Acknowledgements}

The study was supported, in part, by grants from the project "Studio di metodi strumentali per lo screening di individui potenzialmente sottoposti a contaminazione radioattiva - Compagnia San Paolo di Torino".

\section{References}

[1] Pauwels R, Beinsberger J, Collaert B, Theodorakou C, Rogers J, Walker A, , et alThe SEDENTEXCT Project Consortium. Effective dose range for dental cone beam computed tomography scanners. Eur J Radiol 2012;81:267-71.

[2] Roberts JA, Drage NA, Davies J, Thomas DW. Effective dose from cone beam CT examinations in dentistry. Br J Radiol 2009;82:35-40.

[3] Loubele M, Jacobs R, Maes F, Denis K, White S, Coudyzer W, et al. Image quality vs radiation dose of four cone beam computed tomography scanners. Dentomaxillofac Radiol 2008;37:309-18.

[4] Silva MA, Wolf U, Heinicke F, Bumann A, Visser H, Hirsch E. Cone-beam computed tomography for routine orthodontic treatment planning: a radiation dose evaluation. Am J Orthod Dentofacial Orthop 2008;133:640.e1-5.

[5] Lofthag-Hansen S, Thilander-Klang A, Ekestubbe A, Helmrot E, Gröndahl K. Calculating effective dose on a cone beam computed tomography device: 3D Accuitomo and 3D Accuitomo FPD. Dentomaxillofac Radiol 2008;37:72-9.

[6] Ludlow JB, Davies-Ludlow LE, Brooks SL, Howerton WB. Dosimetry of 3 CBCT devices for oral and maxillofacial radiology: CB Mercuray, NewTom 3G and i-CAT. Dentomaxillofac Radiol 2006;35:219-26.

[7] Hashimoto K, Kawashima S, Araki M, Iwai K, Sawada K, Akiyama Y. Comparison of image performance between cone-beam computed tomography for dental use and four-row multidetector helical CT. J Oral Sci 2006:48:27-34.

[8] Loubele M, Bogaerts R, Van Dijck E, Pauwels R, Vanheusden S, Suetens P, et al. Comparison between effective radiation dose of CBCT and MSCT scanners for dentomaxillofacial applications. Eur J Radiol 2009;71:461-8.

[9] Chau AC, Fung K. Comparison of radiation dose for implant imaging using conventional spiral tomography, computed tomography, and cone-beam computed tomography. Oral Surg Oral Med Oral Pathol Oral Radiol Endod 2009;107:559-65.
[10] Bianchi SD, Rampado O, Luberto L, Genovesio AF, Bianchi CC, Ropolo R. Image quality analysis and low dose dental CT. Int Congr Ser 2005;1281:1177-81.

[11] Loubele M, Jacobs R, Maes F, Schutyser F, Debaveye D, Bogaerts R, et al. Radiation dose vs image quality for low-dose CT protocols of the head for maxillofacial surgery and oral implant planning. Radiat Prot Dosimetry 2005; $117: 211-6$

[12] International Commission on Radiological Protection Publication 103. Recommendations of the international commission on radiological protection. Ann ICRP 2007;37:1-332. ICRP publication 103.

[13] Rampado O, Garelli E, Deagostini S, Ropolo R. Dose and energy dependence of response of Gafchromic ${ }^{\circledR}$ XR-QA film for kilovoltage X-ray beams. Phys Med Biol 2006;51:2871-81.

[14] Butson MJ, Cheung T, Yu PK. Measurement of energy dependence for XRCT radiochromic film. Med Phys 2006;33:2923-5.

[15] McKeever SWS, Moscovitch M, Towsend PD. Thermoluminescence dosimetry materials: properties and uses. Ashford, England: Nuclear Technology Publishing; 1995.

[16] Horowitz YS. Study of the annealing characteristics of LiF:Mg, Ti using computerized glow curve deconvolution. Radiat Prot Dosimetry 1990;33: 255-8.

[17] Alva H, Mercado Uribe H, Rodriguez Villafuerte M, Brandan ME. The use of a reflective scanner to study radiochromic film response. Phys Med Bio 2002;47:2925-33.

[18] Thomas G, Chu RY, Rabe F. A study of GafChromic XR Type R film response with reflective-type densitometers and economical flatbed scanners. J Appl Clin Med Phys 2003;4:307-14.

[19] Devic S, Seuntjens J, Sham E, Podgorsak EB, Schmidtlein CR, Kirov AS, et al Precise radiochromic film dosimetry using a flat-bed document scanner. Med Phys 2005;32:2245-53.

[20] Ma CM, Coffey CW, DeWerd LA, Liu C, Nath R, Seltzer SM, et al. AAPM protocol for $40-300 \mathrm{kV}$ x-ray beam dosimetry in radiotherapy and radiobiology. Med Phys 2001;28:868-93.

[21] Tomic N, Devic S, DeBlois F, Seuntjens J. Reference radiochromic film dosimetry in kilovoltage photon beams during CBCT image acquisition. Med Phys 2010;37:1083-92.

[22] Boivin J, Tomic N, Fadlallah B, Deblois F, Devic S. Reference dosimetry during diagnostic CT examination using XR-QA radiochromic film model. Med Phys 2011;38:5119-29.

[23] Huda W, Sandison GA. Estimation of mean organ doses in diagnostic radiology from Rando phantom measurements. Health Phys 1984;47:463-7.

[24] International Commission on Radiological Protection Publication 89. Basic anatomical and physiological data for use in radiological protection: reference values. Ann ICRP 2002;32:5-265.

[25] Rampado O, Garelli E, Ropolo R. Computed tomography dose measurements with radiochromic films and a flatbed scanner. Med Phys 2010;37:189-96.

[26] International Commission on Radiation Unit and Measurements Report 74. Patient dosimetry for X rays used in medical imaging. J ICRU 2005;5.

[27] Struelens L, Vanhavere F, Smans K. Experimental validation of Monte Carlo calculations with a voxelized Rando-Alderson phantom: a study on influence parameters. Phys Med Biol 2008;53:5831-44.

[28] Nunn AA, Davis SD, Micka JA, DeWerd LA. LiF:Mg, Ti TLD response as a function of photon energy for moderately filtered $x$-ray spectra in the range of 20-250 kVp relative to 60Co. Med Phys 2008;35:1859-69.

[29] Zhang D, Savandi AS, Demarco JJ, Cagnon CH, Angel E, Turner AC, et al. Variability of surface and center position radiation dose in MDCT: Monte Carlo simulations using CTDI and anthropomorphic phantoms. Med Phys 2009;36: 1025-38.

[30] Winslow JF, Tien CJ, Hintenlang DE. Organ dose and inherent uncertainty in helical CT dosimetry due to quasiperiodic dose distributions. Med Phys 2011;38:3177-85.

[31] Davies-Ludlow LE, Ludlow JB. Dosimetry comparison of the Newtom $3 G$ and the Newtom VG. In: IADR/AADR/CADR 87th General Session and Exhibition; April 1-4, 2009

[32] Suomalainen A, Vehmas T, Kortesniemi M, Robinson S, Peltola J. Accuracy of linear measurements using dental cone beam and conventional multislice computed tomography. Dentomaxillofac Radiol 2008;37:10-7.

[33] Brady S, Yoshizumi T, Toncheva G, Frush D. Implementation of radiochromic film dosimetry protocol for volumetric dose assessment to various organs during diagnostic CT procedures. Med Phys 2010;37:4782-92. 